\title{
A Robust Linear MPC Approach to Online Generation of 3D Biped Walking Motion
}

\author{
Camille Brasseur*, Alexander Sherikov*, Cyrille Collette ${ }^{\dagger}$, Dimitar Dimitrov* and Pierre-Brice Wieber* \\ *INRIA Rhône-Alpes, \\ 38334 Montbonnot Cedex, France \\ E-mail: \{camille.brasseur, alexander.sherikov, dimitar.dimitrov, pierre-brice.wieber\}@inria.fr \\ $\dagger$ Aldebaran Robotics, Paris, France \\ E-mail: ccolletteealdebaran-robotics.com
}

\begin{abstract}
A crucial part in biped walking motion generation is to ensure dynamic feasibility, which takes the form of a nonlinear constraint in the general case. Our proposition is to bound the nonlinear part of the dynamic feasibility constraint between some properly chosen extreme values. Making sure that this constraint is satisfied for the extreme values guarantees its satisfaction for all possible values in between. This follows a classical approach from robust nonlinear control theory, which is to consider a nonlinear dynamical system as a specific selection of a time-invariant Linear Differential Inclusion. As a result, dynamic feasibility can be imposed by using only linear constraints, which can be included in an efficient linear MPC scheme, to generate 3D walking motions online. Our simulation results show two major achievements: 1) walking motions over uneven ground such as stairs can be generated online, with guaranteed kinematic and dynamic feasibility, 2) walking on flat ground is significantly improved, with a 3D motion of the CoM closely resembling the one observed in humans.
\end{abstract}

\section{INTRODUCTION}

Online generation of biped walking motions in three dimensions, because the ground is not flat, or simply to introduce some desired vertical motion of the body, is still largely an open problem in humanoid robotics today. When approaching this problem, the following questions need to be answered: how can we make sure to generate dynamically and kinematically feasible motions, and then, what are desirable characteristics and objectives for a 3D biped walking motion?

A crucial part in biped walking motion generation is to ensure dynamic feasibility, which takes the form of a nonlinear constraint in the general case. While it is possible to account for this nonlinear constraint in online computations [1], [2], this requires expensive computations which would be better avoided with the limited CPU resources embedded in robots. As a result, various options have been proposed to circumvent this nonlinearity.

The most common approach is to consider the Center of Mass (CoM) of the robot moving on a horizontal plane [3], since in this case, the dynamic feasibility constraint turns into a linear constraint, which can be included then in a linear Model Predictive Control (MPC) scheme to generate walking motions online [4], [5]. However, imposing the CoM to move on a horizontal plane typically leads to unnatural walking motions, with low trunk and bent knees, that require greatly increased torques and excessive speeds in knee joints [6], both impacting negatively the efficiency of the resulting motion in terms of amplitude, speed and energy consumption.

In case the vertical motion of the CoM can be decided before-hand, different linear approaches are possible [7], [8], [9], [10], [11], [12], [13], but having to generate the vertical and horizontal motions of the CoM independently necessarily limits the capacity to deal with 3D objectives and constraints, such as kinematic constraints. We aim here at generating the whole 3D motion in a unified approach.

Our proposition is to bound the nonlinear part of the dynamic feasibility constraint between some adequate extreme values. Making sure that this constraint is satisfied for these extreme values guarantees, as a result, that it is satisfied for all possible values between them. And this involves only linear constraints, which can be included therefore in an efficient linear MPC scheme, to generate 3D walking motions online. This follows a classical approach from robust nonlinear control theory, which is to consider a nonlinear dynamical system as a specific selection of a timeinvariant Linear Differential Inclusion [14], [15]. This will be discussed in Section II.

Concerning kinematic feasibility, many issues such as collision avoidance between the robot and its environment, or between the different parts of the robot, are important but not specific to the problem of walking motion generation, and will not be discussed here. One issue that has to be addressed though, especially in the case of $3 \mathrm{D}$ walking motions, is the limits of the maximal reachable region of the CoM of the robot with respect to the support foot. This maximal reachable region usually has a nonlinear shape, but this shape can be very well approximated by a convex polyhedron, leading to linear constraints that can be included then directly in a linear MPC scheme [11]. This will be briefly discussed in Section III.

By lack of a well-established theory, it is still very unclear today what are exactly desirable characteristics of a 3D biped walking motion, which might depend moreover on the specific mechatronic structure of each robot, and how these characteristics should be formulated within an online motion generation scheme. A popular approach is to follow a mechanical template such as the Spring-Loaded Inverted Pendulum (SLIP) model, originally based on the observation 
of leg stiffness in running animals [16], and later adapted to biped walking on level ground [17]. It is nonlinear and requires complex tuning when generating 3D walking motions [18], but it can also be approximated linearly by considering the spring effect only in the vertical direction [9], [19].

We propose here to follow a much more practical approach. The motivation behind introducing a vertical motion of the CoM is to improve the efficiency of the walking motion in terms of amplitude, speed and energy consumption over potentially uneven terrain. This is mostly done by stretching knees as much as possible, and we will approach this by simply trying to stay as high as possible above the ground, within kinematic constraints [20]. This will be discussed in Section IV.

The complete walking motion generation scheme will be presented in Section V, extending the MPC scheme presented previously in [21]. Finally, simulation results in Section VI will show two major achievements:

1. Walking motions over uneven ground such as stairs can be generated online, with guaranteed kinematic and dynamic feasibility.

2. Walking on flat ground is also significantly improved, with a $3 \mathrm{D}$ motion of the CoM closely resembling the one observed in humans.

\section{DYNAMIC FEASIBILITY CONSTRAINTS}

The most direct approach to the dynamics of robot locomotion starts with the Newton and Euler equations of motion of the whole robot:

$$
\begin{aligned}
m(\ddot{c}+g) & =\sum f_{i}, \\
\dot{L} & =\sum\left(p_{i}-c\right) \times f_{i},
\end{aligned}
$$

which relate the contact forces $f_{i}$ acting on the robot at points $p_{i}$ and the gravity $-g$ to the motion of the CoM of the robot $c$ and its angular momentum $L$ around $c$, with $m$ the mass of the robot. They can be combined in a very standard way (see [22] for details) to obtain:

$$
\frac{m c \times(\ddot{c}+g)+\dot{L}}{m\left(\ddot{c}^{z}+g^{z}\right)}=\frac{\sum p_{i} \times f_{i}}{\sum f_{i}^{z}},
$$

where the superscript ${ }^{z}$ indicates the vertical coordinate of a $3 \mathrm{D}$ vector. We are going to neglect the angular momentum $L$ in the following, as usual when generating standard walking motions [22]. Considering then only the ${ }^{x}$ and ${ }^{y}$ coordinates of the previous equation, we obtain:

$$
c^{x, y}-\frac{c^{z}}{\ddot{c}^{z}+g^{z}}\left(\ddot{c}^{x, y}+g^{x, y}\right)=\frac{\sum p_{i}^{x, y} f_{i}^{z}-p_{i}^{z} f_{i}^{x, y}}{\sum f_{i}^{z}} .
$$

In the MPC scheme proposed in Section V, dynamic feasibility needs to be checked only every $100 \mathrm{~ms}$. By synchronizing this sampling period with the single support phases, we can reasonably assume that, even when walking on uneven ground, dynamic feasibility needs to be checked only at time instances where all contact points are coplanar. Hence, we can choose a different frame for each contact surface in such a way that all the corresponding contact points $p_{i}$ have the same height $p^{z}$. In this case, the previous equation can be rewritten as:

$$
c^{x, y}-\frac{c^{z}-p^{z}}{\ddot{c}^{z}+g^{z}}\left(\ddot{c}^{x, y}+g^{x, y}\right)=\frac{\sum p_{i}^{x, y} f_{i}^{z}}{\sum f_{i}^{z}},
$$

where the right hand side appears to be the Center of Pressure $(\mathrm{CoP})$ of the normal contact forces $f_{i}^{z}$ on the support plane. In the usual case of unilateral contact, with forces $f_{i}^{z} \geq 0$, we end up with the standard constraint that the CoP must lie in the convex hull of contact points (see [22] for details):

$$
c^{x, y}-\frac{c^{z}-p^{z}}{\ddot{c}^{z}+g^{z}} \ddot{c}^{x, y} \in \operatorname{conv}\left\{p_{i}\right\} .
$$

Here and later, we assume without loss of generality the gravity vector to be aligned with the $z$ axis of the frame, i.e., $g^{x, y}=0$. This means that we will consider stepping only on horizontal surfaces here, but this is not a limitation of the proposed approach.

The constraint (6) is linear with respect to the horizontal motion of the $\operatorname{CoM}\left(c^{x, y}\right.$ and $\left.\ddot{c}^{x, y}\right)$, but nonlinear with respect to its vertical motion $\left(c^{z}\right.$ and $\left.\ddot{c}^{z}\right)$. Taking into account this nonlinear model in online computations is possible [1], but this requires more expensive computations, which would be better avoided with the limited CPU resources embedded in robots. As a result, various options have been proposed to circumvent this nonlinearity when generating walking motions online. The most common approach is to consider a CoM moving on a horizontal plane, so that the nonlinear term

$$
\zeta=\frac{c^{z}-p^{z}}{\ddot{c}^{z}+g^{z}}
$$

is constant. In this case, the dynamic feasibility constraint (6) is a constant linear constraint on the horizontal motion of the CoM, which can be included then in a linear MPC scheme [5] to generate walking motions online. The problem is, imposing the CoM to move on a horizontal plane leads to very inefficient motions in terms of amplitude, speed and energy consumption [6].

Another approach is with a vertical motion of the CoM decided before-hand as a function of time. In this case, we obtain a time-varying linear constraint on the horizontal motion of the CoM, which can be considered in a linear MPC scheme [11], in computations based on the Divergent Component of Motion [12], or solved analytically in some limited cases [8]. Another option is to refer to the previous model, with a CoM moving on a horizontal plane, and simply compensate for the difference by iterating the corresponding linear MPC scheme [9], [10]. But having to generate the vertical and horizontal motions of the CoM independently necessarily limits the capacity to deal with 3D objectives and constraints, such as the kinematic constraints discussed in the next Section. We aim here at generating the whole 3D motion in a single computation.

One option then is to resort to the previous model, with a CoM moving on a horizontal plane, and bound the difference 


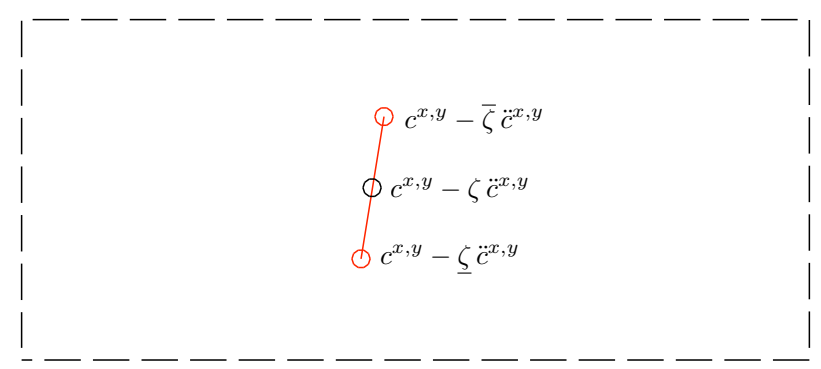

Fig. 1. If we make sure that $c^{x, y}-\zeta \ddot{c}^{x, y}$ and $c^{x, y}-\bar{\zeta} \ddot{c}^{x, y}$ are in the convex hull of the contact points (here the dashed rectangle), then we know that the $\operatorname{CoP} c^{x, y}-\zeta \ddot{c}^{x, y}$, which lies somewhere in-between, also lies in this convex hull, and dynamic feasibility is guaranteed.

with the case when the CoM is moving vertically. This difference would be equal to

$$
\delta=\left(\frac{c^{z}-p^{z}}{\ddot{c}^{z}+g^{z}}-\frac{c_{\text {const }}^{z}-p^{z}}{g^{z}}\right) \ddot{c}^{x, y},
$$

what can be bounded by considering adequate limits on $\ddot{c}^{x, y}$, $\ddot{c}^{z}$ and $c^{z}$, as proposed in [23].

Our proposition here is slightly different. We start by reformulating the dynamic feasibility constraint (6), emphasizing the role of $\zeta$ :

$$
c^{x, y}-\zeta \ddot{c}^{x, y} \in \operatorname{conv}\left\{p_{i}\right\} .
$$

We consider then that variations of $\zeta$ can be reasonably bounded during normal walking motions, as will be verified in the simulations of Section VI:

$$
\underline{\zeta} \leq \zeta \leq \bar{\zeta}
$$

As a result, the CoP appears to always lie on a line segment, between the points $c^{x, y}-\zeta \ddot{c}^{x, y}$ and $c^{x, y}-\bar{\zeta} \ddot{c}^{x, y}$ (see Figure 1). If we make sure that these two points lie in the convex hull of contact points:

$$
\left\{c^{x, y}-\underline{\zeta} \ddot{c}^{x, y}, c^{x, y}-\bar{\zeta} \ddot{c}^{x, y}\right\} \in \operatorname{conv}\left\{p_{i}\right\},
$$

then we know that the $\mathrm{CoP}$ also lies in this convex hull, and dynamic feasibility is guaranteed. Note that the bounds (10) can be enforced as a linear constraint on the vertical motion of the CoM with a simple reformulation:

$$
\underline{\zeta}\left(\ddot{c}^{z}+g^{z}\right) \leq c^{z}-p^{z} \leq \bar{\zeta}\left(\ddot{c}^{z}+g^{z}\right)
$$

$\left(\ddot{c}^{z}+g^{z}=\frac{1}{m} \sum f_{i}^{z}>0\right.$ as long as the robot is not freefalling).

The constraints (11) and (12) are linear constraints on the motion of the CoM, which can be included therefore in a linear MPC scheme, to generate 3D walking motions online. They constrain independently the horizontal and the vertical motions of the CoM, but together, they ensure that the nonlinear dynamic feasibility constraint (6) is satisfied. We will see in the simulations of Section VI, that these constraints provide much tighter bounds on the CoP than (8), and therefore a much more precise evaluation of the dynamic feasibility of 3D walking motions.

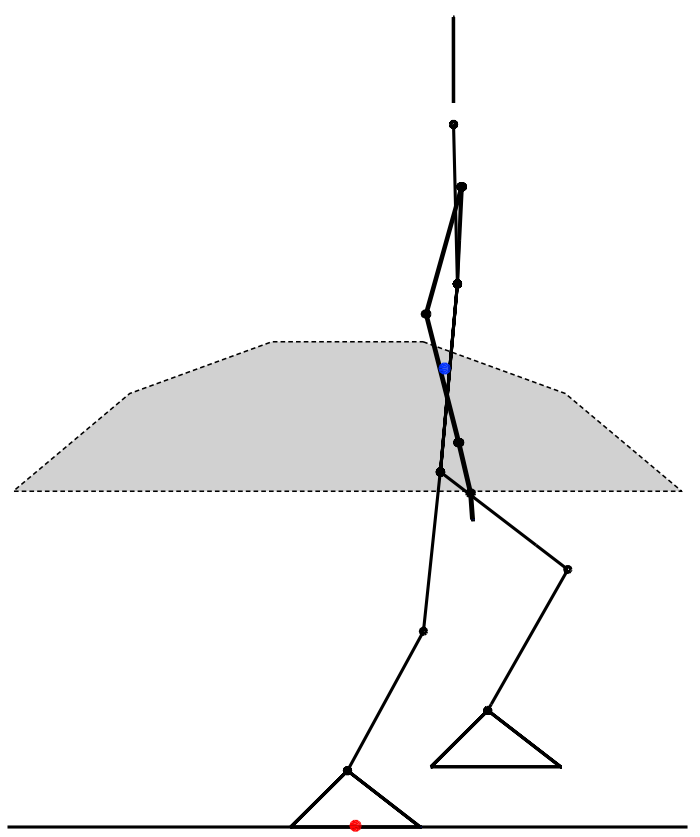

Fig. 2. The maximal reachable region of the CoM of the robot $c$ (blue dot) with respect to the center of the support foot $p$ (red dot) usually has a nonlinear shape, but this shape can be very well approximated by a convex polyhedron, here in gray.

\section{KINEMATIC FEASIBILITY CONSTRAINTS}

In the previous Section, discussing the dynamic feasibility of $3 \mathrm{D}$ walking motions, the focus was on the motion of the CoM $c$ with respect to contact points $p_{i}$. Generating the corresponding joint motions is mostly a question of Inverse Kinematics, which introduces usual kinematic feasibility constraints. Many issues such as collision avoidance between the robot and its environment, or between the different parts of the robot, are important but not specific to the problem of walking motion generation, and will not be discussed here. One issue that has to be addressed though, especially in the case of 3D walking motions is the limits of the maximal reachable region with fully extended legs.

In situations where the $\mathrm{CoM}$ is assumed to move on a horizontal plane, this question is usually approached under the sole view of maximal distance between footprints [21], since in this particular case, stable walking motions do not allow the distance between the $\mathrm{CoM}$ and contact points to grow unchecked. This results in satisfying kinematic feasibility constraints implicitly. Note also that tackling this kinematic limit indirectly requires introducing very conservative approximations.

But when considering vertical motions of the CoM, one of the main objectives is to walk with straighter knees, closer to kinematic limits, which need therefore to be addressed explicitly and precisely. The maximal reachable region of the CoM of the robot $c$ with respect to the center of the support foot $p$ usually has a nonlinear shape, but this shape can be very well approximated by a convex polyhedron (see Figure 2), leading to linear constraints :

$$
A(c-p) \leq b,
$$


with some fixed matrix $A$ and vector $b$. This constraint can be included then directly in a linear MPC scheme [11].

\section{3D WALKING OBJECTIVES}

Walking is a complex task, that needs to satisfy various, potentially conflicting objectives. Unfortunately, by lack of a well-established theory, it is still very unclear today what are exactly the desirable characteristics of a 3D biped walking motion, which might depend moreover on the specific mechatronic structure of each robot, and how these characteristics should be formulated within an online motion generation scheme. A popular approach is to follow a mechanical template such as the Spring-Loaded Inverted Pendulum (SLIP) model, originally based on the observation of leg stiffness in running animals [16], and later adapted to biped walking on level ground [17]. It is nonlinear and requires complex tuning when generating 3D walking motions [18], but it can also be approximated linearly by considering the spring effect only in the vertical direction [9], [19]. We propose here to follow a much more practical approach, simple and robust enough to not require any particularly complex tuning.

The motivation behind introducing a vertical motion of the CoM is to improve the efficiency of the walking motion in terms of amplitude, speed and energy consumption over potentially uneven terrain. This is mostly done by stretching knees as much as possible, what can be approached by simply trying to stay as high as possible above the ground [20], or as close as possible to a high enough reference $h_{\text {ref }}$, minimizing for example the deviation

$$
d_{1}=\left\|c^{z}-\left(p^{z}+h_{r e f}\right)\right\|^{2} .
$$

Regarding the locomotion objective itself, we propose to simply consider following a reference horizontal speed of the CoM $\dot{c}_{r e f}^{x, y}$, minimizing for example [21] the deviation

$$
d_{2}=\left\|\dot{c}^{x, y}-\dot{c}_{r e f}^{x, y}\right\|^{2} .
$$

Robustness of the walking motion with respect to disturbances can be greatly improved by keeping the CoP close to the center $p$ of the support foot [4], [24]. In our case, the $\mathrm{CoP}$ is lying somewhere between the two bounds introduced in (11). We propose therefore to keep the middle of this line segment close to the center of the support foot, minimizing the deviation

$$
d_{3}=\left\|c^{x, y}-\frac{1}{2}(\underline{\zeta}+\bar{\zeta}) \ddot{c}^{x, y}-p^{x, y}\right\|^{2} .
$$

Naturally, it is also important that the generated walking motion is smooth enough to be compatible with the general mechatronic structure of the robot. A usual choice in this respect is to consider that the motion follows a third order dynamics [4], [25], and keep the third derivative small, minimizing for example

$$
d_{4}=\|\dddot{c}\|^{2} .
$$

\section{A LINEAR MPC SCHEME TO GENERATE WALKING MOTIONS ONLINE}

Generating walking motions online naturally implies avoiding to fall in the first place (when possible). This can be modeled as a viability condition [26], [27], and one way to fullfil it is through Model Predictive Control [28]. This is a standard approach to generating walking motions, which can take various forms [22]. Here, since the constraints introduced in the previous Sections are all formulated as linear functions of the motion of the CoM of the robot, and the objectives $d_{1 \ldots 4}$ are all formulated as quadratic functions to minimize, we can consider using a linear MPC scheme, such as the one proposed in [21].

In this MPC scheme, the acceleration $\ddot{c}$ of the CoM is considered to be continuous, piecewise linear (the third derivative $\dddot{c}$ is therefore piecewise constant) over time intervals of constant duration $t_{k+1}-t_{k}=100 \mathrm{~ms}$. At each sampling time $t_{k}$, the constraints and objectives are sampled over the next $N=16$ time intervals, and the following optimization problem is solved:

$$
\begin{aligned}
\operatorname{minimize} & \sum_{j} \alpha_{1} d_{1}\left(c_{j}^{z}\right)+\alpha_{2} d_{2}\left(\dot{c}_{j}^{x, y}\right) \\
& +\alpha_{3} d_{3}\left(c_{j}^{x, y}, \ddot{c}_{j}^{x, y}, p_{j}^{x, y}\right)+\alpha_{4} d_{4}\left(\dddot{c}_{j}\right)
\end{aligned}
$$

subject to (11)-(13) for all $t_{j}$,

with decision variables $\dddot{c}_{j}$ and $p_{j}^{x, y}, j=k, \ldots, k+N$, and positive scalar gains $\alpha_{1 \ldots 4}$ assigned to the different objectives. The third derivative of the CoM $\dddot{c}_{j}$, and the footstep placement $p_{j}^{x, y}$ obtained as a solution to this problem, are applied to the robot during the next time interval, and the problem is solved again at the next sampling time, following a standard MPC procedure [29]. Details on how this optimization problem can be formulated and solved as a standard Quadratic Program can be found in [21].

In this control scheme, the placement of the footsteps $p_{j}^{x, y}$ is decided automatically by the optimization process [21]. Note however that on uneven ground, foot placement will be a nonlinear problem in general, even discontinuous when considering certain obstacles or terrain such as stairs. In order to keep a linear formulation, we limit the approach here to automatic placement within predefined horizontal surfaces. Planning automatically which horizontal surface to use is possible with Mixed Integer Programming [30], but we will consider here that the assignment of each footstep to a specific horizontal surface is decided independently. The automatic footstep placement is allowed then exclusively within the assigned horizontal surfaces. For example, when facing stairs, the automatic footstep placement is allowed only within the limits of the pre-assigned steps.

\section{Simulation RESUlts}

The proposed linear MPC scheme has been tested on a simulated HRP-2 robot [31]. Based on the motion of the CoM and footsteps computed with this scheme, the whole body motion is obtained with the standard inverse dynamics approach and hierarchical optimization [32], [33]. To be 


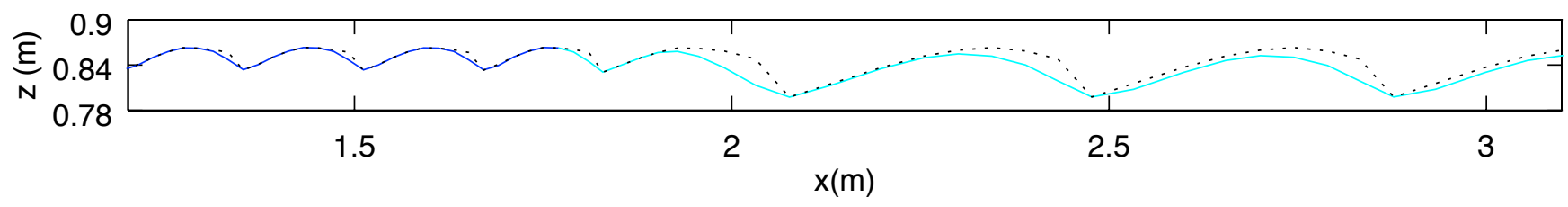

Fig. 3. Trajectory of the CoM of the robot in the sagittal plane when walking on a flat, even ground, transitioning from a speed of 0.2 m.s ${ }^{-1}$ (dark blue) to a speed of $0.6 \mathrm{~m} . \mathrm{s}^{-1}$ (light blue). The dotted curve is the kinematic limit due to maximal leg length. When the step length increases with speed, the maximal CoM height during the double support phase lowers significantly, resulting in a more pronounced vertical motion of the CoM.

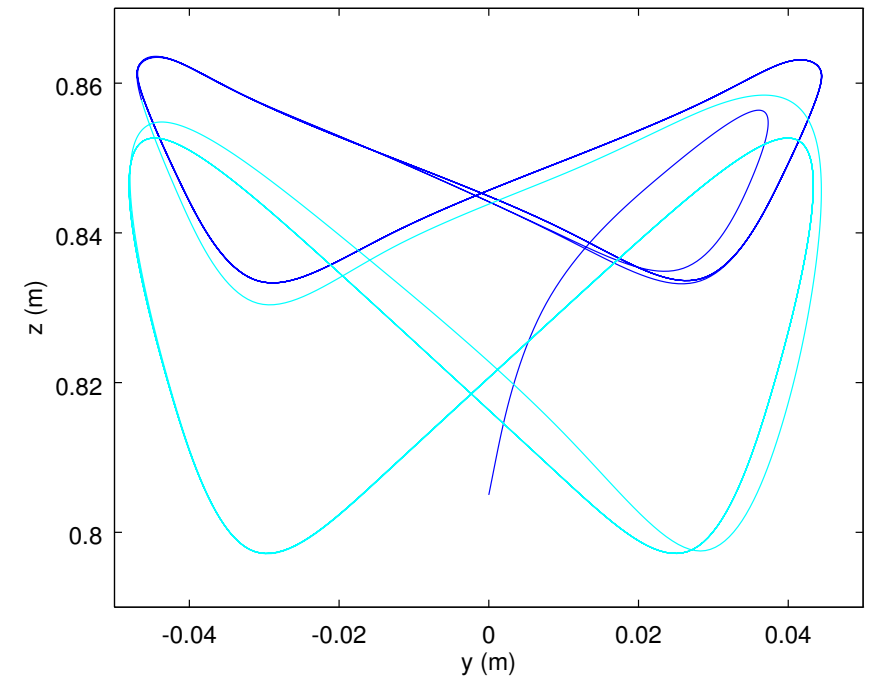

Fig. 4. Trajectory of the CoM of the robot in the frontal plane when walking on a flat, even ground, starting from rest and reaching a speed of $0.2 \mathrm{~m} . \mathrm{s}^{-1}$ (dark blue), then transitioning to a speed of $0.6 \mathrm{~m} . \mathrm{s}^{-1}$ (light blue). The typical butterfly shape and how it changes with walking speed matches what is measured in human walking [34].

more precise, the generated trajectories of the $\mathrm{CoM}$ and feet are tracked by PD-controllers subject to the constraints due to dynamics of the robot, friction, and mechanical limits of the robot, in addition to this, motions of redundant degrees of freedom are damped. Two different simulations are presented.

The first simulation is designed to validate how the proposed scheme behaves on a flat, even ground. It involves starting from rest, walking straight at a (low) speed of $0.2 \mathrm{~m} . \mathrm{s}^{-1}$ for a few steps, then accelerating to a (normal) speed of $0.6 \mathrm{~m} . \mathrm{s}^{-1}$. In the proposed scheme, the timing of the steps is imposed, with a constant period of $0.8 \mathrm{~s}$. As a result, the only way to vary speed is to vary step length, with a direct impact on the kinematic constraints (13) affecting the motion of the CoM.

We can observe in Figure 3 how these kinematic constraints evolve during this simulation, and how the motion of the CoM is adapted accordingly in the sagittal plane. This figure focuses on the transition in walking speed, and includes a few steps before and after. Clearly, when the step length increases with speed, the maximal CoM height during the double support phase lowers significantly, resulting in a more pronounced vertical motion of the CoM. This increase

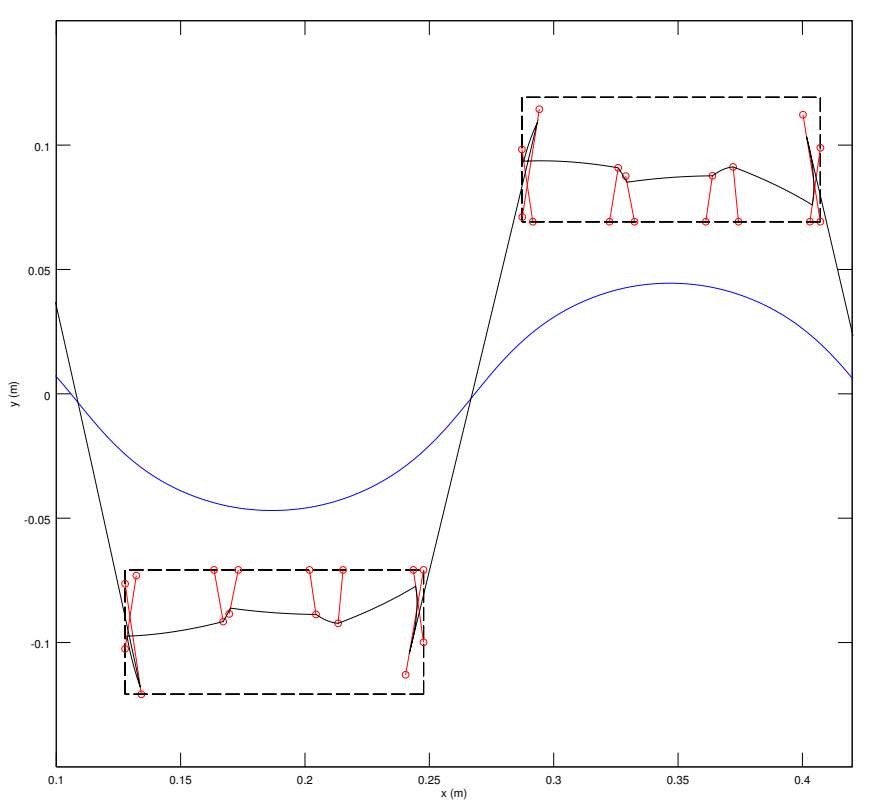

Fig. 5. Trajectories of the CoP (black curve) and CoM of the robot (blue curve), footprints (dashed rectangles), and bounds introduced in Section II (red segments), shown here every $100 \mathrm{~ms}$. We can check that the CoP always stays within these bounds, and that these bounds always stay within the footprints, as desired, guaranteeing the dynamic feasibility of the generated $3 \mathrm{D}$ walking motion.

in vertical amplitude is even clearer in the frontal plane, shown in Figure 4. Here, the trajectory of the CoM presents a typical shape of a butterfly, similar to what can be observed in human walking [34]. We can clearly observe how this curve changes with walking speed, once again similarly to what is measured in human walking [34]. Note also the seamless transitions, from rest, and between walking speeds.

Figure 5 shows the corresponding trajectory of the CoP on the ground during one step. We can verify (here every $100 \mathrm{~ms}$ ) that, as recognized in Section II, the CoP always lies somewhere within the line segment introduced in (11). We can also verify that this line segment stays within the support polygon as desired, satisfying the constraint (11) and guaranteeing as a result the dynamic feasibility of the generated motion.

The largest horizontal and vertical accelerations of the CoM during this walking motion are respectively 2.4 and $4.7 \mathrm{~m} . \mathrm{s}^{-2}$, with the height of the CoM varying between 0.80 and $0.86 \mathrm{~m}$. Under these conditions, the difference (8) between the approximate model with a constant height of 
$0.80 \mathrm{~m}$ and the real position of the CoP can be as large as $6.3 \mathrm{~cm}$, larger than the half-width of the feet $(5 \mathrm{~cm})$. As a result, it is impossible to guarantee with the criterion proposed in [23] the dynamic feasibility of this rather standard motion (which would be discarded on this single account), whereas we can obtain this guarantee here with the much more precise criterion (11).

The second simulation involves climbing up and down stairs, as a typical example of uneven ground. The position and size of the stairs is supposed perfectly known, and the assignment of each footstep to a given stair is supposed decided beforehand (automatic footstep placement is enabled, but within the boundaries of each stair). We can observe in Figure 6 how the vertical motion of the CoM is automatically generated (online) together with its horizontal motion, in order to fit well within kinematic constraints, while ensuring dynamic feasibility throughout the whole motion.

\section{CONCLUSION}

We have proposed a linear MPC scheme for online generation of 3D biped walking motions over flat and uneven ground (assuming that the configuration of the ground is known). It is built on the observation that dynamic feasibility, which takes the form of a nonlinear constraint in the general case, can be ensured with a combination of linear constraints on the horizontal and vertical motions of the CoM of the robot. Simulation results show two major achievements: 1) walking motions over uneven ground such as stairs can be generated online, with guaranteed kinematic and dynamic feasibility, 2) walking on flat ground is also significantly improved, with a 3D motion of the CoM closely resembling the one observed in humans. Experiments with real robots should follow soon.

Note that some important aspects of whole body motion generation have not been approached here. One is the generation of the motion of the feet above the ground, especially avoiding undesired collisions with the environment, as discussed in [10]. Another is a robust Inverse Kinematics scheme, as discussed in [35], since the walking motion generated here approaches kinematic limits, and therefore kinematic singularities. These are necessary additions to the proposed motion generation scheme.

We can also observe that when the robot is walking at $0.6 \mathrm{~m} . \mathrm{s}^{-1}$, the amplitude of the vertical motion of the CoM appears in Figure 4 to be twice as much as the amplitude observed in humans with similar step length and frequency, and similar kinematic structure [34]. Since this amplitude is directly related to kinematic constraints, a possible explanation of this mismatch is the use of toe flexion by humans, which is not mirrored by the proposed motion generation scheme. Toe flexion significantly expands the maximal reachable region obtained with extended legs [6], [11], and could be a promising addition to the proposed scheme, to further improve the general efficiency of the generated walking motion. Another option to expand the maximal reachable region with extended legs, not approached yet in biped robotics, would be to include rotations of the pelvis [36].

\section{ACKNOWLEDGMENTS}

This work has been funded by the PSPC Romeo 2 project and EU H2020 Comanoid Research and Innovation Action (RIA).

\section{REFERENCES}

[1] K. Van Heerden, "Planning COM trajectory with variable height and foot position with reactive stepping for humanoid robots," in Proceedings of the IEEE International Conference on Robotics \& Automation, 2015, pp. 6275-6280.

[2] S. Feng, X. Xinjilefu, W. Huang, and C. Atkeson, "3d walking based on online optimization," in Humanoid Robots (Humanoids), 2013 13th IEEE-RAS International Conference on, Oct 2013, pp. 21-27.

[3] S. Kajita and K. Tani, "Study of dynamic biped locomotion on rugged terrain - derivation and application of the linear inverted pendulum mode -," in Proceedings of the IEEE International Conference on Robotics \& Automation, april 1991, pp. 1405-1411.

[4] S. Kajita, F. Kanehiro, K. Kaneko, K. Fujiwara, K. Harada, K. Yokoi, and $\mathrm{H}$. Hirukawa, "Biped walking pattern generation by using preview control of Zero Moment Point," in Proceedings of the IEEE International Conference on Robotics \& Automation, september 2003, pp. 1620-1626.

[5] P.-B. Wieber, "Trajectory free linear model predictive control for stable walking in the presence of strong perturbations," in Proceedings of the IEEE-RAS International Conference on Humanoid Robots, 2006.

[6] K. Nishiwaki, S. Kagami, Y. Kuniyoshi, M. Inaba, and H. Inoue, "Toe joints that enhance bipedal and fullbody motion of humanoid robots," in Proceedings of the IEEE International Conference on Robotics \& Automation, 2002.

[7] M. Morisawa, S. Kajita, K. Kaneko, K. Harada, F. Kanehiro, K. Fujiwara, and H. Hirukawa, "Pattern generation of biped walking constrained on parametric surface," in Proceedings of the IEEE International Conference on Robotics \& Automation, april 2005, pp. 24162421.

[8] K. Terada and Y. Kuniyoshi, "Online gait planning with dynamical 3D-symmetrization method," in Proceedings of the IEEE-RAS International Conference on Humanoid Robots, 2007.

[9] Z. Li, N. Tsagarakis, D. Caldwell, and B. Vanderborght, "Trajectory generation of straightened knee walking for humanoid robot icub," in International Conference on Control, Automation, Robotics and Vision, 2010.

[10] K. Nishiwaki, J. Chestnutt, and S. Kagami, "Planning and control of a humanoid robot for navigation on uneven multi-scale terrain," in Proceedings of the International Symposium on Experimental Robotics, 2010.

[11] A. Herdt, N. Perrin, and P.-B. Wieber, "LMPC based online generation of more efficient walking motions," in Proceedings of the IEEE-RAS International Conference on Humanoid Robots, 2012.

[12] M. Hopkins, D. Hong, and A. Leonessa, "Humanoid locomotion on uneven terrain using the time-varying divergent component of motion," in Proceedings of the IEEE-RAS International Conference on Humanoid Robots, 2014.

[13] Y. Zhao and L. Sentis, "A three dimensional foot placement planner for locomotion in very rough terrains," in Proceedings of the IEEE-RAS International Conference on Humanoid Robots, 2012.

[14] J.-P. Aubin and A. Cellina, Differential Inclusions. Springer, 1984.

[15] S. Boyd, L. El Ghaoui, E. Feron, and V. Balakrishnan, Linear Matrix Inequalities in System and Control Theory. SIAM, 1994.

[16] R. Full and D. Koditschek, "Templates and anchors: Neuromechanical hypotheses of legged locomotion on land," The Journal of Experimental Biology, vol. 202, pp. 3325-3332, 1999.

[17] H. Geyer, A. Seyfarth, and R. Blickhan, "Compliant leg behaviour explains basic dynamics of walking and running," Proceedings of the Royal Society B, vol. 273, no. 1603, p. 2861, 2006.

[18] Y. Liu, P. Wensing, D. Orin, and Y. Zheng, "Dynamic walking in a humanoid robot based on a $3 \mathrm{~d}$ actuated dual-slip model," in Proceedings of the IEEE International Conference on Robotics \& Automation, 2015, pp. 5710-5717.

[19] I. Mordatch, M. de Lasa, and A. Hertzmann, "Robust physics-based locomotion using low-dimensional planning," in ACM SIGGRAPH Papers, 2010. 


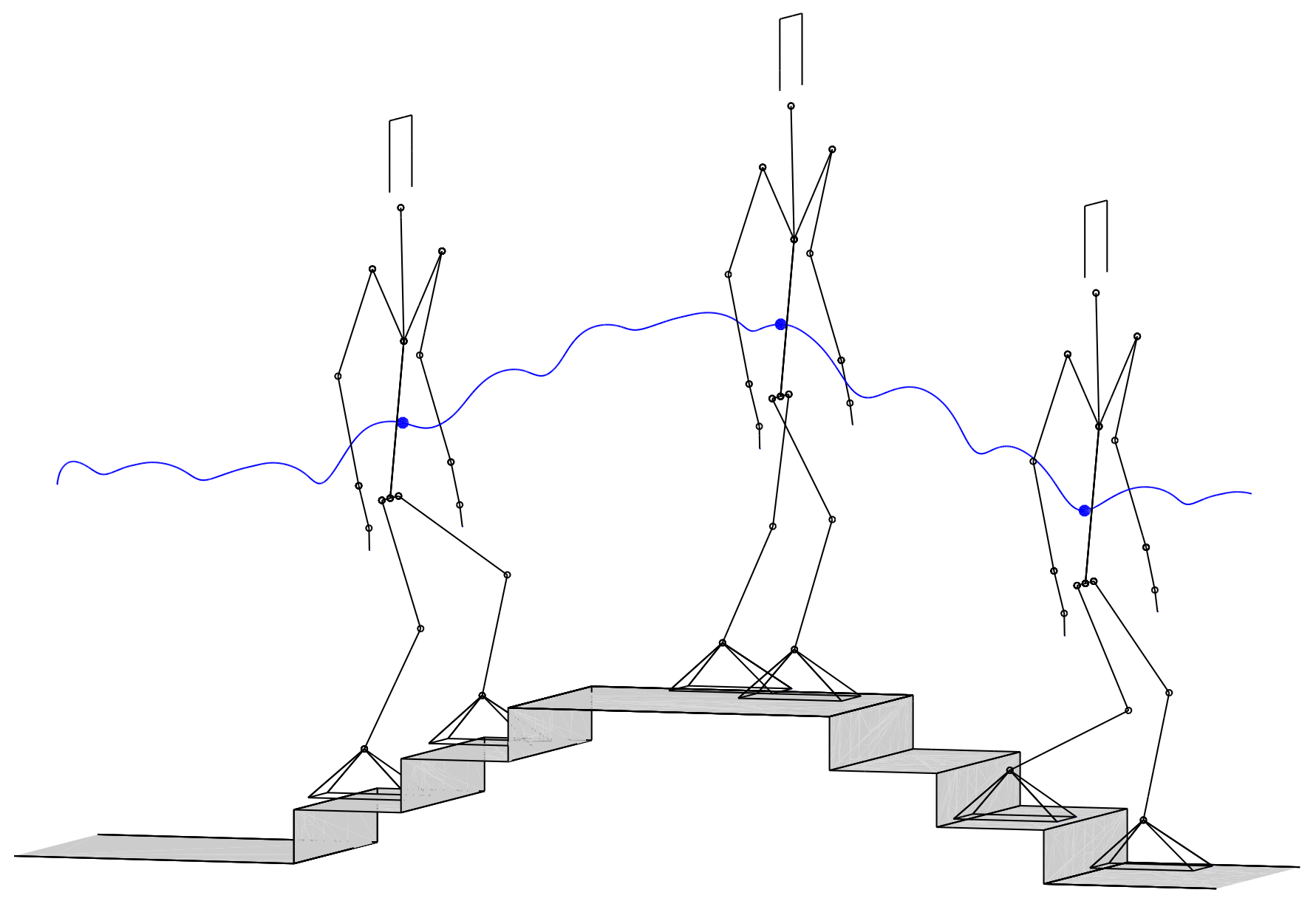

Fig. 6. Snapshots of a walking motion climbing up and down stairs, generated online with the proposed linear MPC scheme. The blue dot is the CoM of the robot, and the blue curve is its trajectory during the motion.

[20] M. Miura, M. Morisawa, F. Kanehiro, S. Kajita, K. Kaneko, and K. Yokoi, "Human-like walking with toe supporting for humanoids," in Proceedings of the IEEE/RSJ International Conference on Intelligent Robots \& Systems, september 2011, pp. 4428-4435.

[21] A. Herdt, N. Perrin, and P.-B. Wieber, "Walking without thinking about it," in Proceedings of the IEEE/RSJ International Conference on Intelligent Robots \& Systems, 2010, pp. 190-195.

[22] P.-B. Wieber, R. Tedrake, and S. Kuindersma, "Modeling and control of legged robots," in Springer Handbook of Robotics, 2nd Edition, B. Siciliano and O. Khatib, Eds., 2016, ch. 48.

[23] K. Hu, C. Ott, and D. Lee, "Online human walking imitation in task and joint space based on quadratic programming," in Proceedings of the IEEE International Conference on Robotics \& Automation, may 2014, pp. 3458-3464.

[24] A. Herdt, H. Diedam, P.-B. Wieber, D. Dimitrov, K. Mombaur, and M. Diehl, "Online walking motion generation with automatic foot step placement," Advanced Robotics, vol. 24, no. 5-6, pp. 719-737, 2010

[25] S. Kajita, K. Miura, M. Morisawa, K. Kaneko, F. Kanehiro, and K. Yokoi, "Evaluation of a stabilizer for biped walk with toe support phase," in Proceedings of the IEEE-RAS International Conference on Humanoid Robots, 2012.

[26] J.-P. Aubin, Viability Theory. Birkhäuser, 1991.

[27] P.-B. Wieber, "Constrained dynamics and parametrized control in biped walking," in Proceedings of the International Symposium on Mathematical Theory of Networks and Systems, 2000.

[28] — "Viability and predictive control for safe locomotion," in Proceedings of the IEEE/RSJ International Conference on Intelligent Robots \& Systems, 2008.
[29] D. Q. Mayne, J. B. Rawlings, C. V. Rao, and P. O. M. Scokaert, "Constrained model predictive control: stability and optimality," $\mathrm{Au}$ tomatica, vol. 26, no. 6, pp. 789-814, 2000.

[30] R. Deits and R. Tedrake, "Footstep planning on uneven terrain with mixed-integer convex optimization," in Proceedings of the IEEE-RAS International Conference on Humanoid Robots, 2014.

[31] K. Kaneko, F. Kanehiro, S. Kajita, H. Hirukawa, T. Kawasaki, M. Hirata, K. Akachi, and T. Isozumi, "Humanoid robot HRP-2," in Proceedings of the IEEE International Conference on Robotics \& Automation, 2004, pp. 1083-1090.

[32] Y. Fujimoto and A. Kawamura, "Proposal of biped walking control based on robust hybrid position/force control," in Proceedings of the IEEE International Conference on Robotics \& Automation, 1996, pp. 2724-2730.

[33] L. Saab, O. E. Ramos, F. Keith, N. Mansard, P. Souères, and J.-Y. Fourquet, "Dynamic whole-body motion generation under rigid contacts and other unilateral constraints," IEEE Transactions on Robotics, vol. 29, no. 2, pp. 346-362, 2013.

[34] M. Orendurff, A. Segal, G. Klute, J. Berge, E. Rohr, and N. Kadel, "The effect of walking speed on center of mass displacement," Journal of Rehabilitation Research \& Development, no. 6A, pp. 829-834, 2004.

[35] K. Tanaka and T. Sugihara, "Dynamically consistent motion design of a humanoid robot even at the limit of kinematics," in Proceedings of the IEEE-RAS International Conference on Humanoid Robots, 2014.

[36] J. Rose and J. Gamble, Human Walking. Williams \& Wilkins, 1994. 